\title{
PERIPHERAL NEUROPATHY (PERIPHERAL NEURITIS) WITH BRONCHIECTASIS
}

\author{
BY \\ J. E. CAUGHEY, R. F. WILSON, AND JOHN BORRIE \\ From the Departments of Medicine and Surgery, Medical School, Otago University, New Zealand
}

(RECEIVED FOR PUBLICATION OCTOBER 21, 1957)

The ill-defined group of conditions comprising the syndrome of peripheral or multiple neuritis led Walshe (1946) to ask for a more precise definition. Elkington (1952) pointed out the advantages of the new term " peripheral neuropathy," as it avoids the suggestion that all the lesions are inflammatory in origin. Haymaker and Kernohan (1949), in a comprehensive study of peripheral neuropathy, found that the clinical picture may range from the slight lesion with peripheral weakness and paraesthesiae to a grave ascending fatal neuronitis involving the central nervous system as seen in the so-called Landry syndrome. They considered that the term "Landry-Guillain-Barré syndrome" should be applied to this group of so-called infective polyneuritis. As the syndrome may involve both the central and the peripheral nervous systems, we prefer the term "neuropathy syndrome," which, in the particular cases described here, refers to a bilateral symmetrical affection of the peripheral nerves of the limbs. This paper presents records of two patients who presented with a neuropathy and were found to have an associated bronchiectatic lesion.

\section{CASe Records}

CASE 1.-A housewife, aged 35 years, consulted one of us (J. E. C.) in August, 1953, on account of weakness and paraesthesiae in the feet and hands. Nine months previously, numbness of both big toes began insidiously and gradually spread to the other toes. Some intermittent "pins and needles" felt on the toes became less and less as time passed. Gradually the numbness advanced around the border of the soles of the feet, up to the ankles, and finally up the legs to the knees. Sensation in the legs faded off from the knees downwards. When the soles of the feet were rubbed after a bath, she had "a distinctly unpleasant feeling." A similar numb feeling on the finger tips had been present for a month. Writing gave no difficulty, but sewing was clumsy. There were episodes of sudden weakness in the hands, and she had a tendency to drop things.

Walking became increasingly difficult on account of a slapping type of gait, especially on uneven ground. The feet and ankles were "oddly stiff and tight" all the time but most of all on movement. The ankles, but not the knees, felt weak.

At the age of 9 years she had severe pneumonia complicated by left basal empyema for which three drainage operations were performed during nine weeks in hospital. A chronic loose paroxysmal cough persisted thereafter, commonly productive of mucopurulent sputum, more profuse and purulent during frequently recurring and prolonged colds, and at times blood-stained. She had frequent attacks of mild pleural pain. Her ill-health seriously interrupted her education. She was comparatively well in her early adult years, but after marriage she again had increasing ill-health. Recurring symptoms of upper respiratory tract infection troubled her for many years, especially latterly, and required treatment. Nephritis followed an uneventful first pregnancy, with the second there was mild toxaemia, and the third and last passed uneventfully.

Effort capacity had not been seriously impaired and she had been able to bring up her family largely without domestic aid. Latterly, there had not been any significant exacerbation or change in respiratory symptoms.

The patient was a well-developed woman, cheerful and co-operative. The fingers were slightly clubbed. The palms and soles were hot and moist. There was some flattening and restriction of movement of the left chest. The trachea was not deviated. The percussion note was slightly impaired over the lower part of the left side of the chest laterally and posteriorly. Breath sounds over the dull area were fainter than elsewhere and were partially masked by medium crepitations. Sputum amounted to about $2 \mathrm{oz} .(60 \mathrm{ml}$.) in a day, and was mucopurulent but not offensive. An old empyema scar was present over the left ninth rib posteriorly.

Her breath was inoffensive. The teeth were natural and in good repair, and her general oral hygiene was satisfactory. Nothing of note was found in the alimentary system, and the liver and the spleen were not palpable. The circulatory system was normal, with a blood pressure of $125 / 70 \mathrm{~mm}$. $\mathrm{Hg}$.

In the nervous system the cranial nerves were intact, and there was little objective evidence of disturbance in the arms and hands. The abdominal reflexes were brisk. All deep reflexes were surpris- 
ingly active except for a depressed left ankle jerk. Little motor weakness was found in the limbs apart from some reduction of power at the ankles and in the toes of both feet, and also some loss in the left arm at the elbow, wrist, and fingers. The sensory changes were significant, for there was loss of all modalities in the finger tips and partial loss of vibration sense up to the wrists. In the feet and lower thirds of the legs vibration sense was absent and there was marked loss of light touch, pain, temperature sense, and two-point discrimination. Sense of position, joint sense, and stereognosis were unimpaired everywhere and there was no ataxia. Tone was diminished in both upper limbs but was unimpaired in the lower.

The following investigations were carried out:

Radiographs. - The chest radiograph showed a deformed left ninth rib posteriorly with cross union to the eighth rib. The trachea was not deviated, although the heart was over to the left, the left hemidiaphragm was considerably raised, and the left costophrenic angle was obliterated. Markings of the basal segments of the left lower lobe were concentrated in a manner consistent with collapse. Several small calcified foci were present towards the apices of both lungs.

Bronchograms.-On the left side complete and satisfactory filling was obtained. Bronchiectatic changes of an advanced degree involved all bronchopulmonary segments of the left lower lobe, except the apical segment, and also the lingular segment of the left upper lobe. The remaining bronchopulmonary segments of the upper lobe appeared to be normal. Filling of the right bronchial tree was complete and satisfactory. No abnormality of any of the right bronchopulmonary segments was demonstrated.

Radiographs of the nasal sinuses showed inflammatory changes in the left maxillary antrum, but were otherwise normal.

Bacteriological Examinations.-The sputum gave a light growth of Micrococcus catarrhalis, coagulasenegative staphylococci, and pneumococci, all being penicillin resistant but sensitive to streptomycin, chlortetracycline, chloramphenicol, and oxytetracycline. Some polymorphs and epithelial cells were seen on direct examination.

Pus from the left maxillary antrum contained a few colonies of $\boldsymbol{H}$. influenzae and diphtheroids, sensitive to penicillin, streptomycin, chlortetracycline, chloramphenicol, and oxytetracycline.

The cerebrospinal fluid was under normal pressure and slightly opalescent. There were no cells. The Pandy test was $2+$, the protein was $122 \mathrm{mg}$. per 100 $\mathrm{ml}$., and the colloidal gold test was 11210000 .

The blood examination revealed a mild hypochromic iron-deficiency type of anaemia. The white cells were normal.

The Mantoux test with 1 T.U. of P.P.D. produced a weal $7 \mathrm{~mm}$. across its long axis in 48 hours. The Congo red test was normal.

Treatment.-From the outset cyanocobalamin, 100 $\mu \mathrm{g}$., was given twice weekly, and vitamin capsules were given three times daily, each containing vitamin A, 2,500 units ; aneurine hydrochloride, $0.5 \mathrm{mg}$. ; ribo $\stackrel{\text { ? }}{+}$ flavine, $0.5 \mathrm{mg}$. ; nicotinamide, $7.5 \mathrm{mg}$.; ascorbic acid읃 $15 \mathrm{mg}$. ; and vitamin D, 300 units. After four months 흠 treatment cyanocobalamin was discontinued.

On September 30,1953, the bronchiectatic left lower lobe and lingular segments were resectedo (J. B.). Both were firmly bound down by vascular? adhesions. Convalescence was prolonged. A post $-\overrightarrow{0}$ operative haematoma which developed on October 6, 1953, was first treated by varidase and aspirationc of blood-stained fluid. As it became infected with coagulase-positive staphylococci resistant to penicillin, $\vec{x}$ streptomycin, chlortetracycline, and oxytetracycline, $\vec{c}$ and sensitive only to chloramphenicol, it was surgically evacuated on October 21, 1953, and treated bycr tube drainage until February 24, 1954, when she was discharged home. On October 14, 1954, the chest? wall sinus was reopened and a small chronic empyemapocket was unroofed. The patient was finally dis- $Z$ charged well on February 9, 1955, and since then has been in good health.

Pathological Report on Resected Specimen.-Thect specimen consisted of the left lower lobe and lingu- $\infty$ lar segment of the left upper lobe. The overlying pleura was shaggy and had been the site of wide-O spread adhesions. The cut surface showed bronchiectasis of the lower lobe with surrounding emphysema. $\bar{O}$ Histological examination revealed dilated bronchiơ lined by a single layer of columnar cells or by normal respiratory epithelium. The bronchial walls consisted of varying amounts of granulation tissue $\frac{0}{3}$ and fibrous tissue infiltrated with lymphocytes, plasma cells, eosinophils, and a few neutrophils. Some of these scarred areas contained remnants of muscle, elastic tissue, cartilage, and mucous glands, but in many bronchi the structure had disappeared. Inter-음 stitial fibrosis was marked and the neighbouring $\underset{\times}{ }$ alveoli were lined by cubical cells and contained an inflammatory exudate of mononuclear cells. Areas $\underline{3}$. of emphysema were also present.

The appearances were those of bronchiectasis and 3 bronchopneumonia.

Progress of Peripheral Neuritis.-On January 28, 1954, there was still some difficulty in walking ando slight ataxia. All arm reflexes were brisker than average. The knee jerks were of average briskness, the $\mathrm{N}$ ankle jerks absent, and the plantar responses flexor. $N$ Sensation was impaired in the lower limbs. Light touch was absent over the feet. Appreciation of pin- $\mathrm{W}$ prick was decreased, and the calves were tender.

On February 11, 1954, there had been someco improvement in ankle jerks and sensation. Calf $\frac{\bar{\Phi}}{\Phi}$ tenderness was decreasing. The patient was walking well.

On March 15, 1954, the dysaesthesias had dis- $\frac{0}{0}$ appeared from the hands and were less prominent $\overrightarrow{\mathbb{D}}$ in the legs. There had been a further return of power, $\frac{\rho}{\mathbb{D}}$ only dorsiflexion of the feet being weak. The ankle $\varrho$ jerks were still absent and the knee jerks reduced. In the legs there was still some reduction of vibration 
sense, of appreciation of sense of position, and of touch. The neurological improvement was such as to justify management away from hospital.

On May 31, 1954, no symptoms were complained of in the hands. The dysaesthesias in the feet extended to a point just above the ankle. Power had considerably increased in the feet and the knee jerks and ankle jerks were just present. Vibration could be appreciated in the legs. A slow but definite improvement was occurring.

When seen on October 17, 1956, she said that her ankles still seemed a little stiff. Occasional tingling in the soles of the feet at night had gradually decreased over the past 18 months. She still felt subjective slightly odd feelings in the soles of the feet when they touched the bedclothes. The previous tendency to dropped feet had cleared completely, though there was still slight stiffness on running, and slight loss of spring in the ankles when climbing stairs. She had been free of symptoms in the upper extremities and face for 18 months.

On examination, the cranial nerves were normal; tone, power, and co-ordination of the upper limbs were normal. There was no disturbance of sensation to touch, heat or cold, pin-prick, or vibration sense, or of position sense and passive movements. Paraesthesiae could not be induced by rubbing the fingers. The deep and abdominal reflexes were normal. In the lower limbs tone, power, and co-ordination were normal. There was no change in appreciation of touch or pin-prick, but it was possible to induce slight paraesthesiae by continued rubbing over the lower two phalanges. The knee jerks were present and the ankle jerks also were present but reduced.

CASE 2.-A retired railway clerk, aged 61 years, was admitted to the Dunedin Hospital on August 30, 1956, from the Neurological Out-patient Department for investigation of a peripheral neuritis syndrome.

After an illness in childhood he had had recurring attacks of bronchitis. Three years before he was first seen he developed pleurisy and lobar pneumonia, and since then a chronic cough, which was not productive of much sputum, had persisted. There had been no haemoptysis. Six months before admission the shock of finding his wife collapsed on the floor and seemingly dead had caused him great emotional disturbance. Since that episode he had been impotent and had complained of weakness of the legs, unsteadiness, and sciatic pain. Over this period also, he had noticed "pins and needles" in the finger tips, in the toes, and in the soles of the feet, which occurred irregularly and were made worse by rubbing the finger tips together and by rubbing the soles of the feet.

He smoked 2 to $3 \mathrm{oz}$. of tobacco a week as cigarettes. He was a well-developed, well-nourished man of sallow complexion. He was emotionally unstable from time to time after admission. His fingers were mildly clubbed, the nails being shiny and a little ridged. No other evidence of pulmonary osteoarthropathy was present.
The trachea and heart were not displaced. Movement of the chest was equal, full, and symmetrical. The lung fields were normally resonant anteriorly, but there was some dullness over both bases as high as the sixth intercostal spaces. Breath sounds were of average intensity, and coarse rales were present at both bases.

The cranial nerves were normal. No wasting or fasciculation of the well-developed upper limbs was found, and tone and co-ordination were normal. Small, fine movements were performed with precision. The deep reflexes were present and equal. There was normal appreciation of pain, heat, cold, touch, position sense, passive movement, and the sense of vibration. "Pins and needles" were felt when the finger tips were rubbed.

The abdominal reflexes were present and equal. The lower extremities were well developed and well nourished. Tone, power, and co-ordination were normal. The deep reflexes were very brisk and equal. Appreciation of touch and pain over the tips of the toes was slightly impaired. On admission rubbing induced paraesthesiae in them. Position sense and passive movement were normally appreciated, but the sense of vibration was lost at the ankles and knees. Romberg's sign was positive. The gait was a little ataxic and on a wider base than normal. The heart was not enlarged or displaced. The sounds were natural. The pulse was normal, and the blood pressure $150 / 64 \mathrm{~mm}$. $\mathrm{Hg}$.

Investigations.-The chest radiograph showed changes in the left lower lung field which were also visible in films taken in 1952 and 1953 . They were attributed to fibrotic changes or pulmonary collapse with pleural thickening following the original infection (pneumonia). Both lung fields also showed evidence of moderate generalized emphysema.

In radiographs of the skull, the pineal body was centrally placed. No abnormality was present in plain views.

Bronchograms of the left lung (September 17, 1956) revealed satisfactory filling of the left bronchial tree. As a result of complete collapse of the left lower lobe the bronchopulmonary segments did not fill. Bronchiectasis was present in both subdivisions of the lingular sub-segment of the left upper lobe. The remaining bronchopulmonary segments of this lobe were normal although over-expanded to fill up the major part of the left hemithorax.

The blood Wassermann reaction and Kahn test were negative.

The cerebrospinal fluid was under normal pressure, contained 5 lymphocytes per c.mm. and $45 \mathrm{mg}$. per $100 \mathrm{ml}$. of protein, and gave a negative Wassermann reaction.

The blood total non-protein nitrogen was $33 \mathrm{mg}$. and the sugar $75 \mathrm{mg}$. per $100 \mathrm{ml}$. The red cells were normal, and the haemoglobin 15.8 g. per $100 \mathrm{ml}$. The leucocytes numbered 12,700 per c.mm., with a neutrophil leucocytosis. 
On bronchoscopy, the larynx, trachea, and carina were normal. At least an ounce of particularly foulsmelling sputum was aspirated from the left bronchial tree, and more was aspirated from the left lower lobe, which was clearly a sump of active chronic bronchiectasis. The mucosa of this lobe was inflamed and hypertrophied and the lumen reduced in diameter by half. The right main bronchus was dry, but a few flakes of sputum were seen on the right lower lobe bronchus. The observation of so much sputum at bronchoscopy when the patient denied having any suggested that he swallowed it.

Bacteriological investigation of the bronchial aspirate gave a heavy growth of coagulasepositive staphylococci on culture, but no tubercle bacilli could be cultured.

Progress and Treatment.-After bronchoscopy and aspiration his symptoms were greatly relieved, his breathing was easier, and he coughed less. As a result of postural drainage and a course of penicillin his general condition improved and the subjective complaints of peripheral paraesthesiae regressed. Because of this improvement, and in view of his age and his own wishes, surgery was not advised, nor was biopsy of a peripheral nerve considered justifiable. On October 4, 1956, he was discharged "relieved" to continue postural drainage and to be observed as an out-patient.

Medical treatment consisted of cyanocobalamin, $100 \mu \mathrm{g}$. weekly, and vitamin capsules, as given to the first patient.

On November 16, 1956, the sensation of " pins and needles" in his hands had cleared up almost completely. Some paraesthesiae were felt over the toes when the feet were hot. His walking, previously clumsy, was much improved. No objective sensory disturbance could be demonstrated, power was normal, and all the reflexes were brisk. He was not depressed. Very little sputum was being coughed up and breathing was much easier.

\section{Discussion}

Both patients presented with a peripheral neuropathy predominantly sensory in type, but in time in Case 1 motor involvement became quite marked, especially in the feet. In Case 2 the evidence of motor involvement was slight. Longstanding pulmonary sepsis with bronchiectasis was found in the course of the investigation of these cases. In both, remission of the peripheral neuropathy occurred after treatment of the pulmonary sepsis. In Case 1 a striking picture of remission and progression, varying with the state of the lung sepsis, indicated a clear association between the sepsis and the neuropathy.

The neuropathy sometimes found with carcinoma of the lung has attracted attention in recent years. The older textbooks refer to carcinoma or carcinomatous cachexia as a cause of polyneuritis, but more recent papers by Denny-Brown (1948 Lennox and Prichard (1950), Brain, Daniel, and Greenfield (1951), and others, have drawn attere tion to this association with carcinoma in varios parts of the body and especially in the lung, which comprises the majority of recorded cases. Clinically the neuropathy may be purely sensory, e.g? in Denny-Brown's two cases, or a combination of sensory and motor as described by Wyburio Mason (1948). Subacute cerebellar degeneration may also occur in some cases.

Aetiology.-A number of factors, both intrinsie and extrinsic, may cause peripheral neuropath e.g., chemical agents, metabolic disorders, and is fective states. Among the latter, Kinnier Wilson (1954) lists septicaemia, typhoid and paratyphoit diphtheria, syphilis, tuberculosis, gonorrhoe: smallpox, measles, scarlatina, influenza, pne monia, malaria, and typhus. Harris (1926) ga $\overrightarrow{\mathrm{ve}}$ an almost identical list of infective causes. Ade unrewarding search has been made of the litera ture of the neurologist and of the chest specialist for accounts of an association between periphera neuropathy and influenza and pneumonia. N\& has a report been found of peripheral neuropath associated with bronchiectasis. Accounts of perif pheral neuropathy associated with chronic lun sepsis are rare, although involvement of the peri pheral nervous system in amyloid disease has beent recognized. Such was a possible basis for the peripheral neuritis in our cases, had there bee other supporting signs.

Peripheral neuropathy with deficiency of vita min-B complex may be conditioned by chron sepsis which increases the vitamin requirement. Our patients were well nourished and had no evis dence of vitamin-B deficiency.

Possibly the same aetiological factors were operative in our cases as are present in those wi carcinoma of the lung. The frequent association of chronic pulmonary sepsis with carcinoma of tho lung suggests that the infection rather than the cancer may be the important aetiological factor but this is unlikely, for the peripheral neuropath may precede clinical and radiological evidence of lung malignancy by months.

Finally, the clue may be found in the paper Waksman and Adams (1955). They induced ap experimental allergic peripheral neuropathy witb the same brain tissue and adjuvants as they used produce experimental allergic encephalitis.

Without the confirmation of suitable path $\AA^{\mathbb{Q}}$ logical material from the nervous system, the basis of the pathology of the neuropathy in the two cases described here can only be speculatic, 
but, on the analogy of the neuropathy occurring with lung cancer and the Landry-Guillain-Barré syndrome, it is probable that the main lesions lie in the dorsal and ventral nerve roots, as there was clear involvement of motor as well as of the sensory function in both cases described.

\section{SUMMARY}

1. Two patients are reported, who presented with a peripheral neuropathy and were found to have chronic bronchiectasis.

2. We can find no record of similar cases.

3. With treatment of the lung sepsis the evidence of peripheral neuropathy receded, suggesting that the aetiological factor was in some way related to the pulmonary sepsis.

\section{REFERENCES}

Brain, W. R., Daniel, P. M., and Greenfield, J. G. (1951). J. Neurol. Psychiat., 14, 59.

Denny-Brown, D. (1948). Ibid., n.s. 11, 73.

Elkington, J. St. C. (1952). Proc. roy. Soc. Med., 45, 661.

Harris, W. (1926). Neuritis and Neuralgia, p.9. Oxford University Press, London.

Haymaker, W., and Kernohan, J. W. (1949). Medicine (Baltimore), $28,59$.

Lennox, B., and Prichard, S. (1950). Quart. J. Med., 19, 97.

Waksman, B. H., and Adams, R. D. (1955). J. exp. Med., 102, 213.

Walshe, F. M. R. (1946). Practitioner, 156, 19.

Wilson, S. A. Kinnier (1954). Neurology, 2nd ed., ed. A. N. Bruce, Vol. 1, p. 316. Butterworth, London.

Wyburn-Mason, R. (1948). Lancet, 1, 203. 